\title{
A Novel Method for Pressure Mapping between Shell Meshes of Varying Geometries and Resolutions ${ }^{\dagger}$
}

\author{
Matthew David Marko \\ Naval Air Warfare Center Aircraft Division, Joint-Base McGuire-Dix-Lakehurst, Lakehurst, NJ 08733, USA; \\ matthew.marko@navy.mil \\ † NAVAIR Public Release 2018-1021 Distribution Statement A-“'Approved for public release; distribution \\ is unlimited".
}

Received: 28 May 2019; Accepted: 11 June 2019; Published: 13 June 2019

check for updates

\begin{abstract}
This manuscript discusses a novel method to map pressure results from one 3D surface shell mesh onto another. This method works independently of the actual pressures, and only focuses on ensuring the surface areas consistently match. By utilizing this approach, the cumulative forces consistently match for all input pressures. This method is demonstrated to work for pressure profiles with precipitous changes in pressures, and with small quadrangular source elements being applied to a mix of large quadrangular and triangular target elements, and the forces at all pressure profiles match remarkably.
\end{abstract}

Keywords: mapping; CFD; CSD; FEA; FSI; pressure; mesh; geometry; shell

\section{Introduction}

Numerical methods [1-5] are among the most important and valuable tools for engineers and scientists today. When an engineering analysis is necessary on an object or domain, and that domain is too complex a shape to be mathematically defined as a simple shape (e.g., a cube, cylinder, or sphere), numerical methods serve to mesh the domain up into clearly defined elements, and the calculating power of a computer can give a realistic answer to the engineering question. One of the most common numerical methods for the study of stresses in a solid is Finite Element Analysis (FEA). Meshed domains are often used in the study of Computational Fluid Dynamics (CFD), an entire field separated from FEA of solids. Of crucial importance in practical engineering is Fluid Solid Interactions (FSI), and, while this is a field in-and-of itself, a combination of CFD and FEA is often used in practical engineering design.

In the studies on the stresses of pressure vessels, water twisters and dynamometers, turbines, airfoils, and countless other applications of fluids and solids, the engineer often uses CFD to model the fluid flow around the incompressible solid, and then uses the pressures at the boundary to study the stresses and strains observed in the solid object. Almost always, the meshed geometry of a CFD domain will vary from the meshed surfaces of an FEA model, and a computational approach to properly map the CFD results onto the FEA boundary is necessary [6-19]. In practical application, it is essential that both the pressure functions match for both meshes, and the cumulative forces in all three dimensions all match for both meshed surfaces. This effort demonstrates a simple and robust algorithm to convert the CFD pressure data into boundary conditions for a different FEA mesh.

There are numerous other efforts to better understand Fluid Solid Interactions (FSI), and interactions between Computational Fluid Dynamics (CFD) and Computational Solid Dynamics (CSD). One such study [20], when using commercial software (ANSYS), approximated a vertical launch tube as a simplified rectangular shape; this was done to validate their novel FSI modeling efforts. Another approach [21] uses the Arbitrary-Lagrangian-Eulerian (ALE) technique, where the 
shell mesh arbitrarily moves based on pre-determined user assumptions; the algorithm loosely couples the two solvers by applying an arbitrarily specified interface boundary conditions at the beginning of each time step, with specific shell cells to represent the FSI shell border. Remeshing is another approach for unstructured FSI, but it is very computationally expensive [22]. Finally, one of the most common approaches [23-25] to map between different shell meshes is to use regression schemes, to develop an equation for the pressure at a given location from a source mesh, and try to match to the target mesh; this approach is realistic, but does not necessarily match the forces perfectly unless one uses very fine meshes.

\section{Set-Up}

Before a final list of the target pressure data can be produced, a transfer matrix must first be generated. This transfer matrix is data on each target element, which source elements will impact the target pressures, and their weighted averages. Each target element pressure can be defined as the summation of several weighted source pressures

$$
P_{T}=\sum_{i=1}^{N} P_{S, i} \cdot A_{S, i}
$$

where $P_{T}(\mathrm{~Pa})$ is the target pressure, $P_{S, i}$ is the pressure of $N$ specific sources $i$, where $i$ is defined by the transfer matrix, and $A_{S, i}$ is the dimensionless weight of each source element's impact on the target. It is absolutely possible for a source to have an impact on more than one target, although in practice the overwhelming majority of the force from one source will go to one target. The purpose of generating a transfer matrix that is exclusively dependent on the geometry is because, in practice, when one conducts a CFD simulation for FSI, it is often with multiple time-steps and therefore a parametric FEA study will be conducted using a series of pressure data for varying time-steps. With the transfer matrix, one can make sure the cumulative forces in all three directions match consistently regardless of the specific CFD pressure profile.

The first step in this mapping algorithm is to define the element surfaces for both the source mesh and the target. This is comprised of matrices of nodes and elements. The node matrices, both the source and the target, have dimensions of $N_{N, S} \cdot 3$ and $N_{N, T} \cdot 3$, where $N_{N, S}$ and $N_{N, T}$ represent the number of source and target nodes. These represent the $X, Y$, and $Z$ coordinates of the nodes, and they are in real or double precision format. There also are two element matrices, of dimensions $N_{E, S} \cdot 5$ and $N_{E, T} \cdot 5$, where $N_{E, S}$ and $N_{E, T}$ represent the number of source and target elements, and they are in integer format. Each row of these two matrices represents a unique element, which is comprised of the number of nodes in the element (three for linear triangles or four for linear quads; this algorithm does not process quadratic element faces), followed by the integer value for the row in the node matrix that contains the $X-Y-Z$ coordinate for the specific node. If there is a triangle element, the fourth node is listed simply as 0 . For example, a quad with coordinates at $(1,1,0),(1,2,1),(2,1,0)$, and $(2,2,1)$ would be listed in the element matrix as

whereas the first four nodes of the node matrix would be

$$
\begin{array}{lll}
1.0 & 1.0 & 0.0 \\
1.0 & 2.0 & 1.0 \\
2.0 & 1.0 & 0.0 \\
2.0 & 2.0 & 1.0 .
\end{array}
$$


While each element declared will be used in the generation of the transfer matrix, there can be excess nodes in the node matrix without any detriment, so long as all of the nodes referenced by the elements are present.

The next step is to determine the centroid of each element, for linear tetrahedral faces (triangles) and linear hexahedrals faces (quads); this is simply calculated by taking the $X, Y$, and $Z$ coordinate values of the three or four nodes that comprise the element. Each element row has three columns, regardless of the shape of the element face, for the $X, Y$, and $Z$ coordinates of the centroid of each element. The centroid is necessary for applying a smoothing function in between elements, which is directly related to the distance between centroids. A separate centroid matrix is assigned for both the source and the target elements, with the dimensions of $N_{E, S} \cdot 3$ and $N_{E, T} \cdot 3$.

The next step is to calculate the area of each element face. This is performed by taking the cross product of the vectors between the element node and the center of the triangle. If the element is a triangle, it is split into three mini-triangles formed with the vectors from the node to the centroid. The steps are as follows:

- Find the centroid of the triangle element, and in the case of an element being a quad, the triangle that forms half of the quad element surface

- If a quad, then the first three node elements are used to calculate the area. Afterwards, the process is repeated with the first, second, and fourth node. Regardless of the node order, this should create two triangles that form the quad.

- The centroid coordinates are found by simply averaging the $X, Y$, and $Z$ locations of the three nodes that made up the surface triangle.

- Generate the vectors from each node to the centroid.

- $\quad$ Find the area formed by each of the three pairs of vectors three times.

- $\quad R_{1} \times R_{2}, R_{2} \times R_{3}$, and $R_{1} \times R_{3}$, where $R_{1}, R_{2}$, and $R_{3}$ represent the vector formed in between the centroid and node 1,2 , and 3 , respectively.

- The area is calculated as half of the absolute value of the cross product of the two vectors, to form each smaller triangle

$$
\begin{aligned}
A & =\frac{1}{2} \cdot\left|R_{1} \times R_{2}\right| \\
& =\frac{1}{2} \cdot\left\{\left(R_{1 B} \cdot R_{2 C}-R_{1 C} \cdot R_{2 B}\right)^{2}+\left(R_{1 A} \cdot R_{2 C}-R_{1 C} \cdot R_{2 A}\right)^{2}+\left(R_{1 B} \cdot R_{2 A}-R_{1 A} \cdot R_{2 B}\right)^{2}\right\},
\end{aligned}
$$

where $R_{1}$ and $R_{2}$ are vectors of components $A, B$, and $C$.

- The areas of these three small triangles is added up to determine the area of the larger triangle (Figure 1)

- If the element surface is a quad, this process is performed twice, treating the quad as two triangles

- Different software packages use different node patterns. If the wrong pattern is used, the area could be erroneously calculated (Figure 2),

- It is not an option to find the node the greatest distance away, as an oddly shaped element would give an erroneous calculation (Figure 3),

- The areas of all three combinations of two possible triangles are calculated (Table 1),

- The maximum of these three possible areas is selected as the area of the quad of interest. 


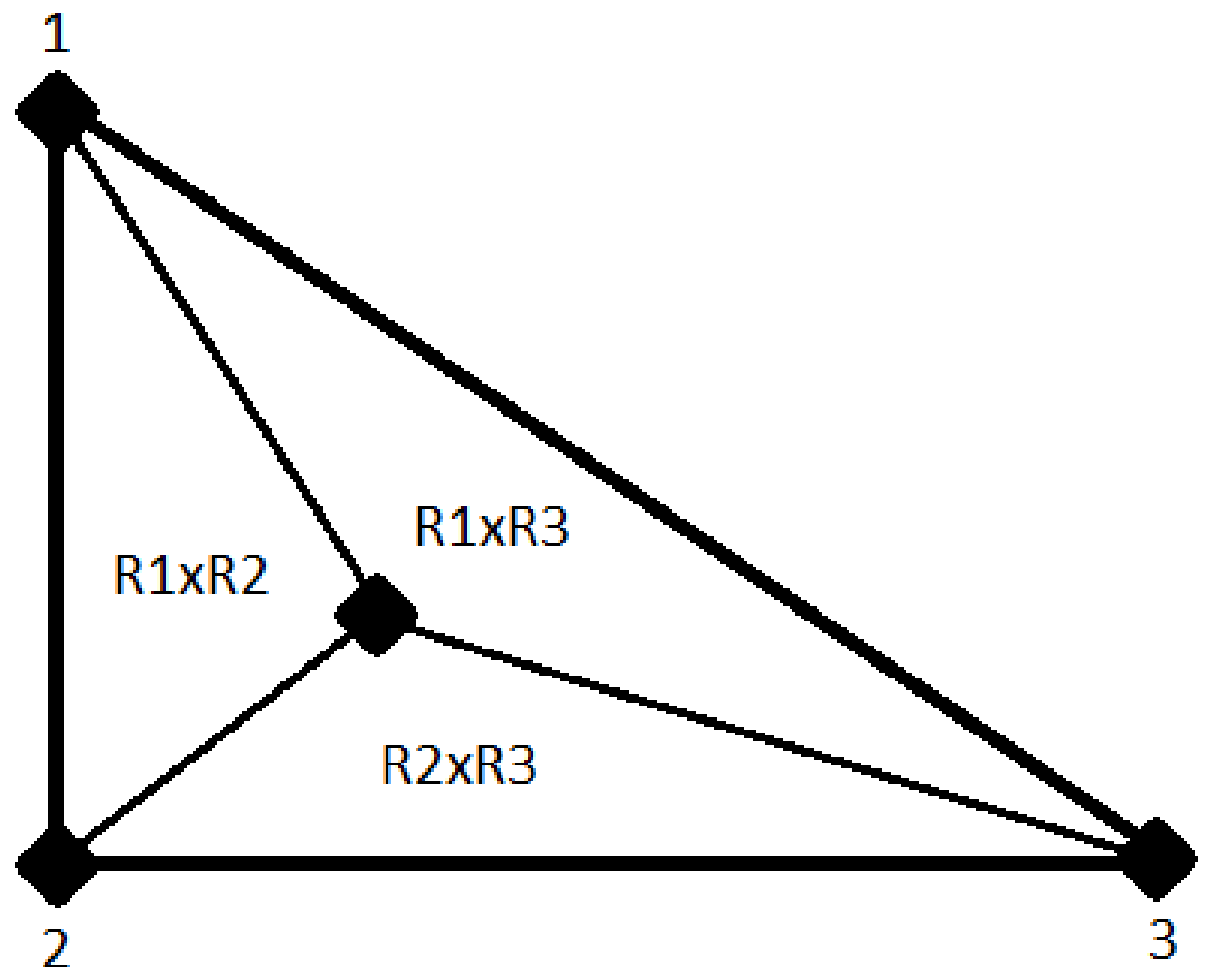

Figure 1. Triangle element face divided into three parts.

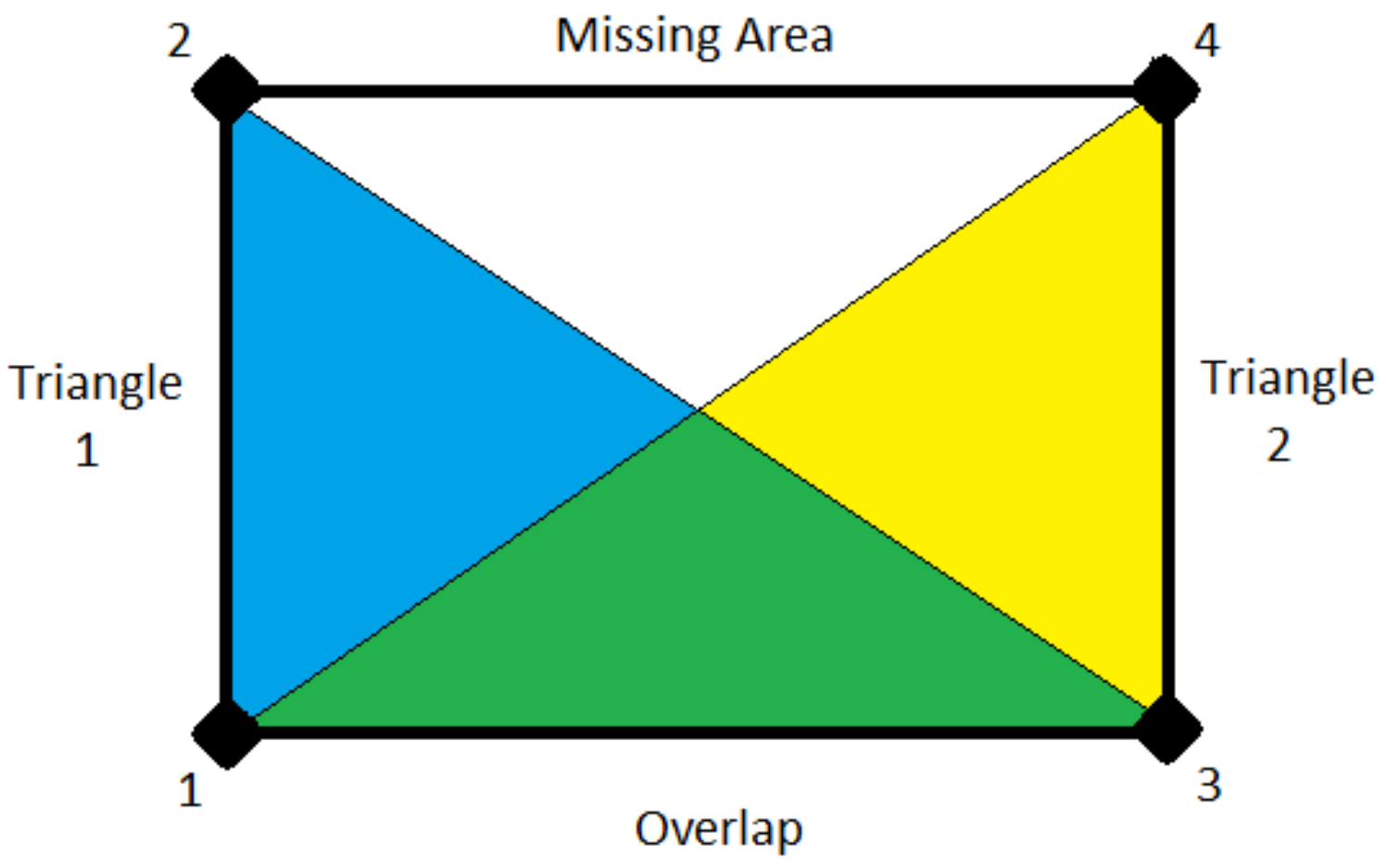

Figure 2. An example of how picking the wrong nodes to divide a quad into two triangles (blue and yellow) can cause error due to area overlap (green), utilizing nodes 1-2-3 and 1-3-4. 


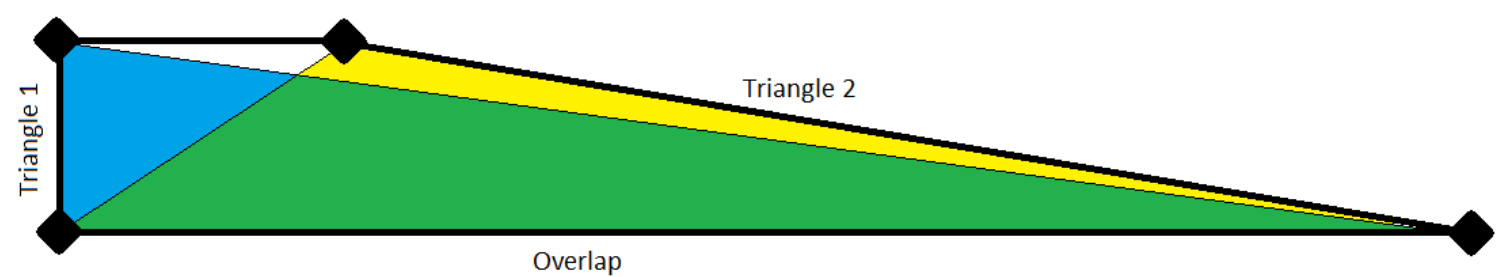

Figure 3. Another example of how picking the wrong nodes to divide a quad into two triangles (blue and yellow) can cause error due to area overlap (green), demonstrating how an assumption cannot be made by searching for the longest vector between two nodes.

Table 1. Three different areas of two triangles; the maximum total area represents the correct area of the quad element face.

\begin{tabular}{ccc}
\hline Quad Area & Node (First Triangle) & Node (Second Triangle) \\
\hline Area 1 & Node $1,2,3$ & Node $1,2,4$ \\
\hline Area 2 & Node $1,3,2$ & Node $1,3,4$ \\
\hline Area 3 & Node $1,4,2$ & Node $1,4,3$ \\
\hline
\end{tabular}

The next step is to calculate the normal vector of each element. In addition to weighting the source-to-target impact by distance between centroid, this approach also considers the angles of each surface. If a source surface is parallel to a target surface, then the force may be fully applied; if the elements' surfaces are perpendicular, then there is no impact of the source on the target. The normal vector is found by normalizing the cross product of the vectors between Node 1-2 and Node 1-3. It would not matter if the element surface was a quad or a triangle, or what order the nodes were located; assuming the surface is flat as would be the case for a linear element, then any combination of node vectors should yield the unit normal.

\section{Algorithm}

Once it is defined what the centroid, surface area, and unit normal vector is for each source and target element, the mapping algorithm can commence. The goal of mapping is to determine how much of an impact each source element will have on each target element. If there is perfect overlap from a source and an element, then all of the force from that source element will be applied to that target element. Of course, in practice, this almost never happens, and therefore a weighting scheme is needed to determine what proportion of each source element is applied to each target element. The impact of a source onto a target can be defined as $W$, a dimensionless number from 0 to 1 ; a value of 0 means there is no force from the source applied to the target, and a value of 1 means all of the force from the source is applied to the target.

The first step is to look at the distance from centroid to centroid, and the relative impact $W_{r}$. A magnitude function should be one where the magnitude decreases exponentially with distance, such as the Gaussian function

$$
W_{r}=\exp \left(-\frac{r}{h}\right),
$$

where $r(\mathrm{~m})$ is the linear distance from the source centroid to the target centroid, and $h(\mathrm{~m})$ is the smoothing value. The value should be such that $W_{r}$ is small if the distance between centroids is so great that the source element is completely outside of the target element. It was found that good results came from setting $h$ to equal the mean average value of the square root of the target element areas. 
The second aspect of the impact $W_{A}$ between source and target elements is the angle separating the unit normal for each element. This value can be found by taking the dot product of the unit normal vectors:

$$
W_{A}=V_{S} \cdot V_{T}=V_{S, x} \cdot V_{T, x}+V_{S, y} \cdot V_{T, y}+V_{S, z} \cdot V_{T, z}
$$

where $V_{S}$ and $V_{T}$ are the unit-normal vectors for the source and the target element. If the two elements are perfectly parallel, then $W_{A}=1$. Even if the centroids of a target element are proximate, if they face perpendicular to each other they ought to not have any impact with each other and $W_{A}=0$. In practical application, if the surfaces are identical, proximate elements should be very close to parallel to each other; only distant source elements might be significantly less parallel, and they will have a low value of $W_{r}$ so that the value of $W_{A}$ should be irrelevant. The importance of this step, however, is when there are sharp edges in a 3D, model; such source elements might have dramatically different directions and therefore should have little impact, yet be proximate to a target element. For this reason, both the separation distance as well as the normal vectors of each source and target vectors are considered to get a total dimensionless weighting value

$$
W=W_{r} \cdot W_{A}
$$

where $W$, a dimensionless value between 0 and 1 , is the weighted impact value of a source and a target.

This approach is performed for source and target pair, and is the most computationally intensive task of the mapper, requiring $N_{S} \cdot N_{T}$ distance calculations, where $N_{S}$ and $N_{T}$ are the number of sources and targets. For typical models of hundreds of thousands of surface elements, an $N_{S} \cdot N_{T}$ array would take up far more memory than most computers can handle, and therefore an optimizing approach is necessary. The first step is categorizing the targets that each source can have a meaningful amount of impact with; this is stored in two $N_{S} \cdot N_{X}$ arrays, where $N_{X}$ is an arbitrarily small, user defined integer value (typically 5 to 10). The first array defined as a real variable array of the impact value $W$; the second is defined as an integer array and represents the target the specific source impacts. For each source element, the impact magnitude of all of the targets was found. To keep the number of contacted targets within the value of $N_{X}$, the calculated impact magnitude was only stored in the array if the value was greater than the minimum of all $N_{X}$ impact magnitudes stored. If it was greater than the minimum, the calculated value was stored in the array location of the previous minimum impact value; the target element number was stored in the equivalent location in the integer array. This method serves the goal of finding the top $N_{X}$ target elements that have the greatest impact magnitude $W$ to a given source.

One potential issue that can come up with mapping is the potential for target elements to have no proximate source elements. This is especially possible from different geometries or meshes. A user may prefer to map every target with the nearest mesh, regardless of how far it is; this will, however, result in pressures not actually applied on the target being predicted. Another option is for the user to set a minimum value of the impact magnitude $W$. This has the advantage of preventing pressures far from a target element from impacting the final pressure calculation; there is the risk (possibly desired by the user) that some target elements fail to get meshed by any source elements. There is another advantage that, since the vast majority of target elements will be too far from the source element of interest to have an impact magnitude $W$ exceed the minimum value of $W$, all these calculated values are not written to memory, significantly reducing run time for large meshes.

After this step has been completed, each of the $N_{S}$ source elements have $N_{X}$ or less target elements with a varying impact magnitude of $W$ ranging from 0 to 1 . The calculated value of $W$ is fairly arbitrary, and the smoothing function can be modified by the user. What cannot be arbitrarily altered is the 
total summation of the forces; the total forces must match in the transfer matrix. With each source, the actual impact of all $N_{X}$ proximate target elements are normalized $W_{N}$, so that

$$
\Sigma_{i=1}^{N_{X}} W_{N, i}=1
$$

These normalized impacts represent the proportion that the force from one source element is applied to a given target element. This approach enables that the total force from each source is equally applied to $N_{X}$ target elements, and the cumulative forces match for both the source and the target.

The transfer matrix does not work in terms of force but of pressure; the goal of the mapper is to find an even distribution of source pressures that combine to a given target element. To determine the magnitude of a source pressure on a target element $W_{P}$, the ratio of the element areas are applied, and thus

$$
W_{P}=W_{N, i} \cdot \frac{A_{S}}{A_{T, i}},
$$

where $A_{S}$ is the area of the source, $A_{T, i}$ is the area of the specific target, $W_{N, i}$ is the normalized ratio of the force from the source that is applied to target $i$, and $W_{P}$ is the ratio of how much pressure from the source is added to the total pressure of target $i$.

The last and final step is to swap the calculated values of $W_{P}$ from a $N_{S} \cdot N_{X}$ array to a $N_{T} \cdot N_{X 2}$ array, where $N_{X 2}$ is an integer value significantly greater than $N_{X}$. This is simply determined by

- $\quad$ Sorting through the $N_{S} \cdot N_{X}$ array of $W_{P}$ for each source element,

- Adding to the target number found, the location in a $N_{T} \cdot N_{X 2}$ array,

- That source element, the source element number, and the pressure magnitude data $W_{P}$.

- Incrementing the count of sources proximate to a given target

- Stored in a separate integer vector $\left(N_{T} \cdot 1\right)$,

- Used to track how many source elements are proximate to the given target element,

- Can be expected to exceed $N_{X}$ provided the number of source elements is greater than the number of target elements,

- Should never exceed $N_{X 2}$; if this happens, it is necessary to increase the value of $N_{X 2}$ to avoid memory errors,

- Save the transfer matrix.

Saving the transfer matrix is the last and final step. This algorithm saves the transfer matrix as a separate output file for the combination of two specific meshes; the actual pressure is irrelevant. The transfer matrix by definition is the proportion of all the source pressures that make up each target pressure, so that the cumulative forces all match. The purpose of saving this transfer matrix is so that, in the future, a host of different pressures can be mapped onto the surface without needing to rerun the computationally expensive mapping script. In practice, a numerical analysis will run in many time-steps, and one might want to map a host of different pressures to the same mesh. The format of the output transfer matrix includes:

- Number of elements $N_{T}$,

- This number is saved so a future mapping algorithm knows the number of target pressures to generate,

- For $N_{T}$ times, list the specific target and the following information,

- $\quad$ Target number, Number of Sources Proximate, and Sum of Pressure Impacts $W_{P}$ 
- The reason for the sum of $W_{P}$ is because each individual value of $W_{P}$ is normalized by the cumulative sum of $W_{P}$ for all proximate targets, so that the pressure of the target element is a proportional average of these proximate source elements.

- For each target, for the number of proximate source elements,

- Sequential number, source element number, and pressure impact $W_{P}$

\section{Test Example}

To demonstrate the robustness of this mapping algorithm, an arbitrary mapped source mesh will be mapped onto a target, for 180 different pressures; the details of this code are described in the Appendix A, and included in the Supplementary file. The source is a grid of 450.450 nodes, spanning from -0.1 to 1.1 in the $X, Y$, and $Z$ direction; the grid is slanted along the $Z$ plane so that $Z=Y$. All of the elements are the quads formed by the 202,500 nodes, resulting in $(450-1)^{2}=201,601$ source elements. The target domain is smaller, stretching from 0 to 1 in the $X, Y$, and $Z$ direction, also parallel to the $Z$ plane, and these nodes are an even grid of $225 \cdot 225=50,625$ nodes. Different from the source, which uses exclusively quad surface elements, the target has a mix of quads and triangles. Moving up in the $Y$ and $Z$ direction, odd numbered rows are all quads, whereas even numbered rows are all triangles, with two triangle surface elements formed per four nodes.

An arbitrary pressure was applied to 180 time steps, all with different pressures. The pressure for a given time step is

$$
P(x, y)=\left(C_{X} \cdot x x^{2}\right)+\left(C_{Y} \cdot y y^{2}\right)+P_{\text {spot }}
$$

where

$$
\begin{gathered}
x x=x+\left(\frac{o o}{180} \cdot 1.2\right), \\
y y=y+\left(\frac{o o}{180} \cdot 1.2\right), \\
C_{X}=500+2 \cdot 00, \\
C_{Y}=1500-3 \cdot 00,
\end{gathered}
$$

and $o o$ is the time step, ranging from 1 to 180. If $x x$ or $y y$ ever exceeded the value of 1.1, then $x x$ or $y y$ was subtracted by 1.2. This will cause a moving pressure boundary in the $X$ and $Y$ direction, demonstrating the robustness for sudden pressure changes. In addition, a Gaussian pressure spike is added at a cyclic location

$$
\begin{array}{r}
P_{\text {spot }}=5000 \cdot \exp (-10 \cdot r r), \\
r r=\sqrt{\left(x-X_{C}\right)^{2}+\left(y-Y_{C}\right)^{2}},
\end{array}
$$

where

$$
\begin{aligned}
& X_{C}=0.5+0.25 \cdot \cos \left(\frac{00 \cdot \pi}{90}\right), \\
& Y_{C}=0.5+0.35 \cdot \sin \left(\frac{o 0 \cdot \pi}{90}\right) .
\end{aligned}
$$

The mapping was completed, and, for all 180 time steps, the cumulative forces matched almost exactly (Figure 4). The forces shown in Figure 4, representing the integrated pressures over area such as in Figure 5, are of arbitrary units; this example uses pressures in Pascals, applied over a surface area of one square meter, to give the force in Newtons. Obviously, the total forces for the source is greater, as the surface area for the source is greater, but, by isolating all of the elements within the range of 
0 to 1 meter that will be applied to the target mesh, the target force matches nearly perfectly for all of the pressure steps.

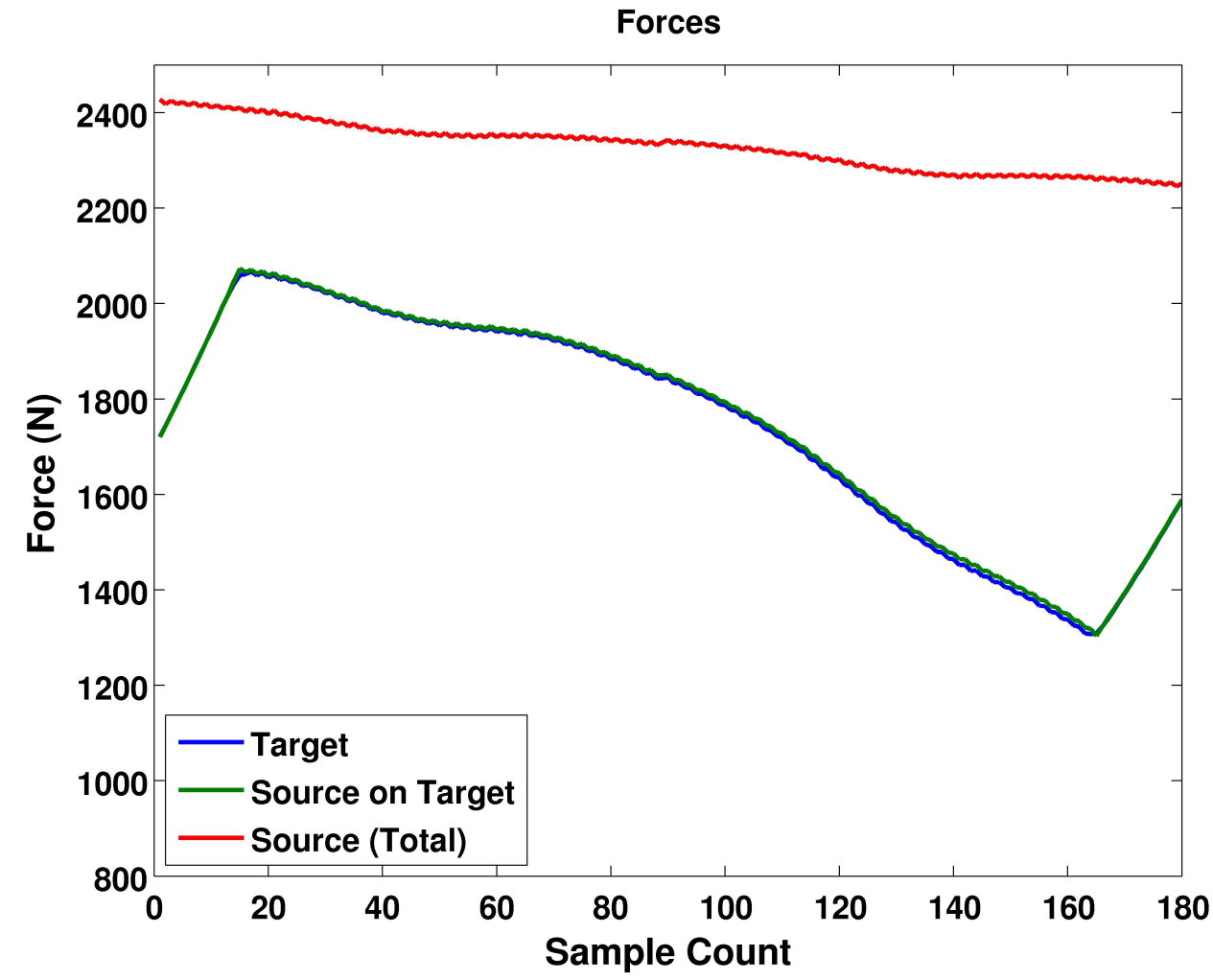

Figure 4. Comparison of the Total Forces in Newtons of the source versus the target, for 180 different source pressure profiles.

When looking at the individual mapped surfaces such as in Figure 5, it is clear that sharp lines, high peaks, and random pressure spikes are unaffected, and the mapped pressures on the target closely match the mapped pressures on the source.

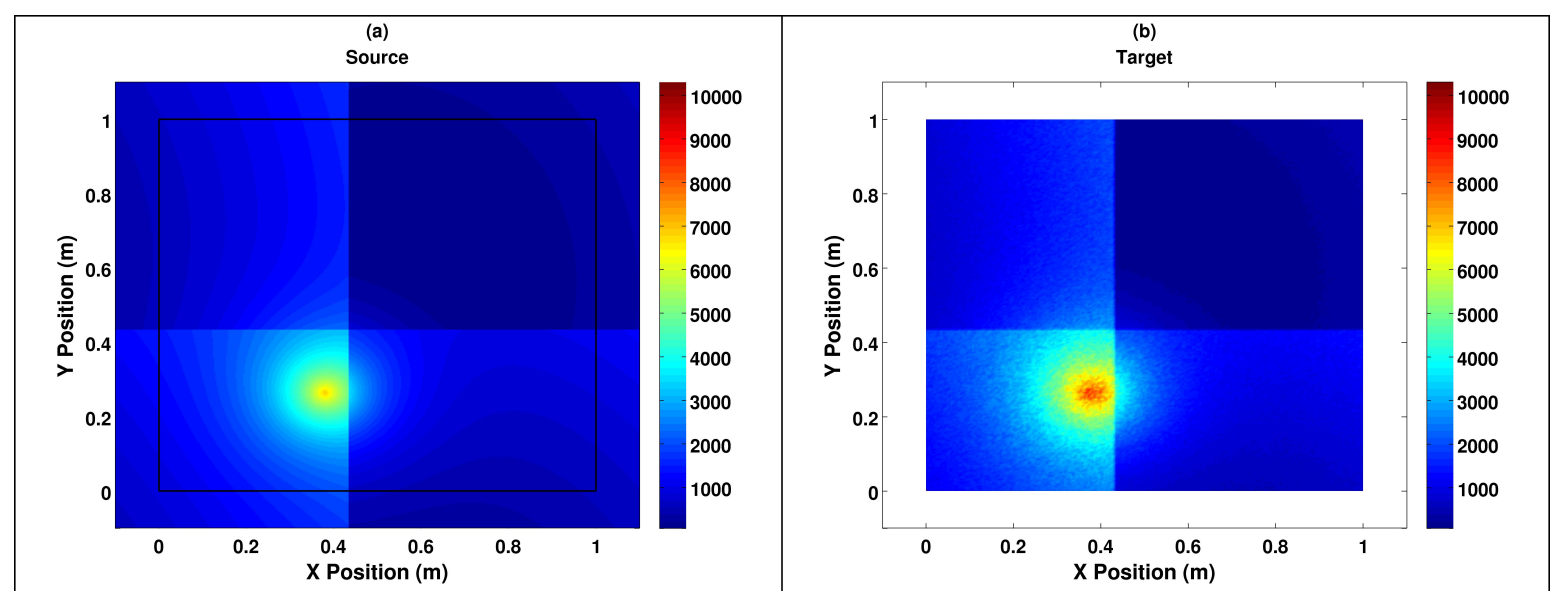

Figure 5. Comparison of the Pressures of one time step, both Source and Target, for oo $=100$. The color-bar represents the pressure $(\mathrm{Pa})$. 


\section{Conclusions}

A robust algorithm has been demonstrated to map pressures from a source shell mesh onto a surface target of a similar geometry but of a different mesh. This algorithm has been tested to work with both quads and triangles, and both geometries work. After mapping two dramatically different meshes, with different overall geometries, different structured elements, and significantly different sizes, forces and pressure profiles have managed to consistently match. This test demonstrates the effectiveness of using this method to robustly map pressures from one mesh onto another.

Supplementary Materials: All sample codes are available online at http://www.mdpi.com/2079-3197/7/2/29/s1. Author Contributions: M.M. is the sole author of this manuscript.

Funding: This research was funded by NAVAIR.

Conflicts of Interest: The author declares no conflict of interest.

\section{Abbreviations}

The following abbreviations are used in this manuscript:

$\begin{array}{ll}\text { ALE } & \text { Arbitrary-Lagrangian-Eulerian } \\ \text { FSI } & \text { Fluid Solid Interactions } \\ \text { CFD } & \text { Computational Fluid Dynamics } \\ \text { CSD } & \text { Computational Solid Dynamics } \\ \text { FEA } & \text { Finite Element Analysis } \\ \text { ANSYS } & \text { Analysis Systems, Inc. } \\ \text { NAVAIR } & \text { Naval Air Systems Command }\end{array}$

\section{Appendix A. Sample Code}

\section{Appendix A.1. Introduction}

This sample code was prepared to demonstrate the mapping test example discussed in the manuscript, where 180 time steps of pressure data are mapped from one mesh to another, all the while preserving the overall forces. This supplementary code enables the users within the Fortran and the MatLab programming environment to recreate the results from start to finish. The steps are as follows: generating the source and target mesh, as well as the input target pressures for 180 time steps; generating the transfer matrix; mapping the input source pressures to generate output target pressures; and post-processing to generate the images and check the total forces on the source and target surfaces. All of these steps are run independent of each other, allowing a future user to customize it as desired.

\section{Appendix A.2. Generating the Input Files}

The first step is to generate the source and target mesh, as well as the source input pressure files. A script to generate all of the sample mesh and pressure files was written in the Fortran programing language as file Make_Input.f and can easily be run after compiling. If one were to run it in the Linux operating system environment with gfortran installed, it can easily be run by typing:

gfortran -03 -o makeinput Make_Input.f

./makeinput

in the command prompt. This assumes that the empty directory Input that is in the supplementary folder remains. In addition, the executable filename makeinput is arbitrary, and can be substituted for any filename the user wishes. Generating the meshes and pressure files should take approximately five minutes on a standard personal computer.

Four files will be generated in the directory that the executable is run: the source node Ns_dat_source.txt and element Es_dat_source.txt input file, as well as the target node Ns_dat_target.txt 
and element Es_dat_target.txt input file. The node files are the $X, Y, Z$ coordinates of each node, and the element files lists which node comprises each triangle or quadrangular element. In addition, 180 input pressures are generated as Ps_001.txt, ...,Ps_180.txt in the input directory are generated. With these files, the mapping algorithm can be compiled and executed in order to get the transfer matrix.

\section{Appendix A.3. Generating the Transfer Matrix}

The second step is to generate the transfer matrix; this file is labeled transfer_matrix.dat. The source input pressure files are not needed at this time; the transfer matrix is only defined by the geometries of the two meshes. It is necessary that in the same directory as the executable file are the source node Ns_dat_source.txt and element Es_dat_source.txt input file, as well as the target node Ns_dat_target.txt and element Es_dat_target.txt input file. Finally, it is necessary to have the input mapper parameter file Map_Parameters.txt in the same directory. This file enables manipulation by the user of the mapping parameters without ever having to manipulate the source make_tm.f Fortran file.

The input mapper parameter file contains six lines of simple inputs; no variable names are shown. The variables in terms of line numbers are as follows:

1. $N x=5$ : This is the maximum number of targets that can be considered proximate to a given source. Matrix arrays of $N s \cdot N x$ will be generated, so it must be small enough that the script does not exceed the memory resources of the computer. Even if there is a lot of memory, unless the target elements are smaller than the source (definitely possible but often not the case in practice), then it should not be desirable for $N x$ to be greater than a few, as one would not want the source element to be mapped onto elements a significant distance away.

2. $N x 2=500$ : This is the maximum number of sources that can be considered proximate to a given target and used in the transfer matrix. Matrix arrays of $N t \cdot N x$ will be generated, so it must be small enough that the script does not exceed the memory resources of the computer, but also large enough to contain every source matrix considered proximate to a given target; in practice, having a value of $N x 2$ that is 100 times greater than $N x$ was found to work well.

3. MinW0 $=0.0000000001$ : This is the minimum value of $W$ that is necessary for a target particle to be considered proximate to a source. This value can be set to zero, but the computational time will be dramatically increased, and there is a risk that source elements that do not in fact overlap a target can be used for the final pressure. If a minimum value is set, then target elements that do not have any proximate source elements will not be mapped, resulting in a consistent pressure of zero. Different circumstances may prefer or oppose this possibility. This value of $10^{-10}$ was used in the example in the manuscript.

4. $h c o e f=1.0$ : This is a coefficient to calculate the weighting function between a source and target element centroid as a function of distance apart. The smoothing length is proportional to this arbitrary value times the square root of the average area of all of the target elements. The larger hcoef is, the more likely a source element fully separated from a target element will influence the pressure, and the less likely a target element will turn out to not be mapped.

5. $\quad c t 1=1$ : This value is not used in the mapping, only in the generation of the final target pressure output files. This fifth line is the number of the first of the input pressures, and should be 1 unless modified by the user.

6. $c t 2=180$ : This value is not used in the mapping, only in the generation of the final target pressure output files. This sixth line is the number of inputs, and should be 180 to capture all 180 input pressures unless modified by the user.

To run the script in the Linux operating system environment, merely enter the following commands

gfortran -03 -o run make_tm.f

./run

in the command line. The executable filename run is arbitrary, and can be substituted for any filename the user wishes. On a typical personal computer, the mesh example generated will take approximately 
an hour and a half to run to completion. Progress on the run is saved in the file prompt.dat and the user can see how many steps of searching remain, as well as the run time to date, by opening this file with the command:

less prompt.dat.

\section{Appendix A.4. Generating New Pressures}

After the transfer matrix is generated, the next step is to map the input pressure files to new output pressure files Pt_001.txt, ...,Pt_180.txt in the empty directory target. There is a simple and practical reason for keeping this script to generate the output pressure files separate from the mapping algorithm to generate the transfer matrix. Quite simply, the transfer matrix, which is computationally expensive to generate, might be used for different analysis, and one very well may reuse a transfer matrix for the same geometry but with different pressure inputs. By separating these steps, this script becomes a practical tool useful in real engineering design.

In the file Map_Parameters.txt the fifth and sixth line are 1 and 180, representing the numbered filename. The choice to use 180 pressure files in this example is arbitrary, merely representing a single revolution (the pressure data was generated with sinusoidal functions) in two-degree increments. There are infinite possible quantities of pressure files that a parametric numerical analysis could generate, depending on the needs and computational resources of the user. To execute the read-map script, merely enter the following commands in the command prompt:

gfortran -03 -o readmap ReadMap.f

./readmap.

The executable filename readmap is arbitrary, and can be substituted for any filename the user wishes. On a typical personal computer, this will take approximately five minutes to run to completion.

\section{Appendix A.5. Post-Processing}

At this step, the new pressures are generated for the target elements, and the forces should match for all pressure steps. To validate and visualize this, a series of MatLab scripts were developed to process and plot the results. The first step is to calculate the total force on the surface; this is easily done by multiplying the pressure times each element's area and summing these forces up:

$$
F=\sum_{i=1}^{N e} P_{i} \cdot A_{i}
$$

When comparing forces, it is necessary to distinguish the force from the source over the $1 \cdot 1$ area of the target, versus the elements on the edge. The next step is to generate the two manuscript figure files, including the cumulation of the forces for all 180 pressure steps in Figure 4, as well as the source and target 2D pressure plots (pressure file 100) in Figure 5. Finally, all of the 2D pressure plots, both source and target are generated and saved, and an animation of the entire change in pressure clearly matches for all time steps. This post-processing script validates the pressure data, and validates that the forces match for all pressure functions, even with dramatic and precipitous shifts in pressure magnitude, and demonstrates that this algorithm is a practical tool to convert pressure data from different 3D surface meshes for numerical analysis in practical engineering design.

\section{References}

1. Haberman, R. Applied Partial Differential Equations with Fourier Series and Boundary Value Problems, 4th ed.; Prentice Hall: Upper Saddle River, NJ, USA, 2003.

2. Nagel, R.K.; Saff, E.B.; Snider, A.D. Fundamentals of Differential Equations, 5th ed.; Addison Wesley: Boston, MA, USA, 1999.

3. Garcia, A. Numerical Methods for Physics, 2nd ed.; Addison-Wesley: Boston, MA, USA, 1999.

4. White, F. Fluid Mechanics, 5th ed.; McGraw-Hill: Boston, MA, USA, 2003. 
5. Cengel, Y. Heat Transfer, a Practical Approach, 2nd ed.; Mcgraw-Hill: New York, NY, USA, 2002.

6. Banerjee, D.K. Software Independent Data Mapping Tool for Structural Fire Analysis. NIST Tech. Note 2014, $1828,1-45$.

7. Smith, W.G.; Ebert, M.P. A Method for Unstructured Mesh-to-Mesh Interpolation; Hydromechanics Department Report; NSWCCD-50-TR-2010; Naval Surface Warfare Center Carderock Division: Bethesda, MD, USA, 2010; p. 056.

8. Tang, T. Moving Mesh Methods for Computational Fluid Dynamics. Contemp. Math. 2005, 383. [CrossRef]

9. Budd, C.J.; Huang, W.; Russell, R.D. Adaptivity with Moving Grids. Acta Numer. 2009, 18, 111-241. [CrossRef]

10. Gao, Q.; Zhang, S. Moving Mesh Strategies of Adaptive Methods for Solving Nonlinear Partial Differential Equations. Algorithms 2016, 9, 86. [CrossRef]

11. Budd, C.J.; Williams, J.F. Moving Mesh Generation Using the Parabolic Monge-Ampere Equation. SIAM J. Sci. Comput. 2009, 31, 3438-3465. [CrossRef]

12. Wang, W.; Yan, Y. Strongly coupling of partitioned fluid-solid interaction solvers using reduced-order models. Appl. Math. Model. 2010, 34, 3817-3830. [CrossRef]

13. Basting, S.; Quaini, A.; Canic, S.; Glowinski, R. Extended ALE Method for fluid-structure interaction problems with large structural displacements. J. Comput. Phys. 2017, 331, 312-336. [CrossRef]

14. Farhat, C.; van der Zee, K.G.; Geuzaine, P. Provably second-order time-accurate loosely-coupled solution algorithms for transient nonlinear computational aeroelasticity. Comput. Methods Appl. Mech. Eng. 2006, 195, 1973-2001. [CrossRef]

15. Degroote, J.; Haelterman, R.; Annerel, S.; Bruggeman, P.; Vierendeels, J. Performance of partitioned procedures in fluid-structure interaction. Comput. Struct. 2010, 88, 446-457. [CrossRef]

16. Wick, T. Fluid-structure interactions using different mesh motion techniques. Comput. Struct. 2011, 89, 1456-1467. [CrossRef]

17. Amsallam, D.; Farhat, C. An Online Method for Interpolating Linear Parametric Reduced-Order Models. SIAM J. Sci. Comput. 2011, 33, 2169-2198. [CrossRef]

18. Amsallem, D.; Zahr, M.; Choi, Y.; Farhat, C. Design optimization using hyper-reduced-order models. Struct. Multidiscip. Optim. 2015, 51, 919-940. [CrossRef]

19. Assessment of Computational Fluid Dynamics (CFD) for Nuclear Reactor Safety Problems; NEA/CSNI/R(2007)13 OECD Nuclear Energy Agency: Le Seine Saint-Germain-12, boulevard des Illesm F-92130 Issy-les-Moulineaux, France, January 2008. Available online: https:/ /inis.iaea.org/search/search.aspx?orig $\mathrm{q}=\mathrm{RN}: 44037880$ (accessed on 11 June 2019)

20. Lee, Y.; Gwak, M.C.; Cho, H.; Joo, H.S.; Shin, S.J.; Yo, J.J.; Shin, J.C. Numerical Simulation of Fluid Structure Interaction Problem Associated with Vertical Launching System. J. Spacecr. Rockets 2018, 55, 948-958. [CrossRef]

21. Cirak, F.; Radovitzky, R. A Lagrangian Eulerian shell fluid coupling algorithm based on level sets. Comput. Struct. 2005, 83, 491-498. [CrossRef]

22. Tremel, U.; Sorensen, K.A.; Hitzel, S.; Rieger, H.; Hassan, O.; Weatherill, N.P. Parallel remeshing of unstructured volume grids for CFD applications. Int. J. Numer. Methods Fluids 2007, 53, 1361-1379. [CrossRef]

23. You, Y.H.; Na, D.; Jung, S.N. Data Transfer Schemes in Rotorcraft Fluid-Structure Interaction Predictions. Hindawi Int. J. Aerosp. Eng. 2018, 2018, 3426237. [CrossRef]

24. Samareh, J.A. Discrete Data Transfer Technique for Fluid-Structure Interaction. In Proceedings of the 18th American Institute of Aeronautics and Astronautics Computational Fluids Dynamics Conference, Miami, FL, USA, 25-28 June 2007; Session: CFD-17: Fluid-Structure Interaction. [CrossRef]

25. Guruswamy, G.P. A review of numerical fluids/structures interface methods for computations using high-fidelity equations. Comput. Struct. 2002, 80, 31-41. [CrossRef]

(C) 2019 by the author. Licensee MDPI, Basel, Switzerland. This article is an open access article distributed under the terms and conditions of the Creative Commons Attribution (CC BY) license (http:/ / creativecommons.org/licenses/by/4.0/). 